\section{UNA EXPERIENCIA SOBRE LA EVALUACIÓN AUTÓNOMA O PARTICIPATIVA: AUTOEVALUACIÓN Y EVALUACIÓN POR LOS COMPAÑEROS}

\author{
Miguel Á. Acedo \\ Francisco J. Ruiz-Cabestre \\ Departamento de Economía y Empresa, Universidad de La Rioja, \\ C/ La cigüeña 60 (Edif. Quintiliano), 26004 Logroño (La Rioja) \\ miguel-angel.acedo@unirioja.es \\ javier.ruiz@unirioja.es
}

\begin{abstract}
This work shows the experience of the implementation of autonomous or participative assessment applied to students of the Degree in Administration and Business Management. Through this experience we try to understand how students perceive their participation in the evaluation process, differentiated between self assessment and peer assessment. We also try to see the degree of objectivity that have the marks obtained through self assessment and peer assessment compared to those given by the lecturer. The results of the experience are relevant and show that autonomous or participative assessment should be considered as a technique in order to assess the learning process.
\end{abstract}

KEY WORDS: Learning assessment; participative assessment; autonomous assessment; self assessment; peer assessment; higher education.

\section{INTRODUCCIÓN}

La evaluación consiste en medir en qué grado el alumno ha alcanzado las competencias (conocimientos, habilidades o destrezas, y actitudes y valores) que se persiguen a lo largo del proceso de enseñanza-aprendizaje de una o varias materias (Gil y Padilla, 2009). Esta tarea de evaluación, basada en el esquema tradicional de la enseñanza universitaria, se ha entendido como una labor inherente al profesor y se centra fundamentalmente en el examen escrito al final del período de docencia, donde lo que se mide principalmente son conocimientos y existe muy poco margen para aprender de los errores.

El nuevo marco del Espacio Europeo de Educación Superior (EEES), que supone un cambio de rumbo importante, al

\section{AN EXPERIENCE ABOUT AUTONOMOUS OR PARTICIPATIVE ASSESSMENT: SELF ASSESSMENT AND PEER ASSESSMENT}

RESUMEN: El presente trabajo muestra la experiencia de la puesta en práctica de la evaluación autónoma o participativa aplicada a los alumnos de LADE en un intento de conocer cómo perciben su participación en el proceso de evaluación, diferenciado entre autoevaluación y evaluación por los compañeros, y qué grado de objetividad tienen las calificaciones obtenidas a través de la autoevaluación y la evaluación por los compañeros frente a las otorgadas por el profesor. Los resultados de la experiencia revelan que la evaluación autónoma o participativa debería ser tenida en cuenta como una técnica e instrumento de evaluación más al servicio de la evaluación del aprendizaje.

PALABRAS CLAVE: Evaluación del aprendizaje; evaluación participativa; evaluación autónoma; autoevalución; evaluación por compañeros; educación superior.

trasladar el centro de gravedad de este proceso desde la enseñanza al aprendizaje, permite plantear una serie de cambios importantes en la evaluación (Bretones, 2008; Calderón y Escalera, 2008; Pérez et al., 2008 y Gessa y Rabadán, 2009). Las cuestiones para qué evaluar, qué evaluar, cuándo evaluar y quién evalúa admiten respuestas más amplias desde la óptica de la evaluación del aprendizaje (Brown, 2003).

Los cambios que deben guiar la evaluación del aprendizaje necesariamente estarán encaminados a determinar el nivel logrado por el alumno en la adquisición de las competencias propias (profesionales y generales) de la materia o materias (Padilla y Gil, 2008). Sin embargo, será imprescindible delimitar las competencias, definir los criterios de desempeño (resultados esperados en los alumnos como 
consecuencia de las competencias adquiridas), recoger evidencias del desempeño (que demuestran la adquisición de las competencias por parte del alumno), analizar las evidencias para juzgar el aprendizaje logrado y comunicar los resultados a los alumnos para orientar y mejorar el proceso de aprendizaje. Para ello, la evaluación deberá estar presente a lo largo de todo el proceso a través de diferentes técnicas e instrumentos de evaluación y podrá ser llevada a cabo por el profesor, el propio alumno y los compañeros (Gil y Padilla, 2009).

En este contexto, se presenta la actual experiencia docente de evaluación, consistente en la puesta en práctica de la evaluación autónoma para las actividades libres de la asignatura de Dirección Financiera II de $3 .^{\circ}$ de LADE. El alumno que se acoge al programa de trabajo de la asignatura, que conlleva la evaluación continua, debe elaborar a lo largo del curso 2 actividades libres por bloques de temas (en concreto, al finalizar los temas 2 y 5), que pueden consistir en cuestiones, reflexiones, problemas y/o pequeños casos libremente diseñados. Estas actividades representan el $15 \%$ de la calificación total y son objeto de autoevaluación y evaluación por los compañeros con la supervisión del profesor, lo que representa una novedad en el proceso de enseñanza-aprendizaje de la asignatura de Dirección Financiera II al involucrar al alumno en una parte de dicho proceso como es la evaluación (Brew, 2003; Jordan, 2003; y Webster y Lapaham, 2003).

Por otro lado, la autoevaluación y la evaluación por los compañeros, además de permitir la evaluación de las competencias profesionales, fomentan y potencian otras competencias generales tales como el "desarrollo de una visión crítica y reflexiva", la "toma de decisiones" y la "asunción de responsabilidades", sin olvidar los valores de "justicia y compromiso" que subyacen en los procesos de evaluación entre iguales, con lo que se facilita la integración del alumno en el proceso de enseñanza-aprendizaje (Gil y Padilla, 2009).

Como resultado de la presente experiencia, se pretende conocer cómo los alumnos perciben su participación en el proceso de evaluación y qué grado de objetividad presentan las calificaciones obtenidas a través de la autoevaluación y la evaluación por los compañeros frente a las otorgadas por el profesor. Para ello, los alumnos han dado respuesta a una encuesta de 16 items (8 de autoevaluación y otros 8 de evaluación por los compañeros), que trata de valorar su percepción ante ambas experiencias. Posteriormente, mediante los oportunos contrastes paramétricos y no paramétricos, se han identificado las diferencias entre la autoevaluación y la evaluación por los compañeros, y, por último, se han comparado las calificaciones obtenidas en la autoevaluación, la evaluación por los compañeros y la evaluación otorgada por el profesor.

En lo que resta el trabajo se estructura en las siguientes secciones. En la segunda se describe la puesta en práctica de la evaluación autónoma. La tercera analiza cómo los alumnos han valorado su participación en dicha experiencia y qué grado de objetividad presentan las calificaciones obtenidas. Y, por último, la cuarta sintetiza las conclusiones más relevantes.

\section{Aplicación de la evaluación autónoma: AUTOEVALUACIÓN Y EVALUACIÓN POR LOS COMPAÑEROS}

La puesta en práctica de la evaluación autónoma, se ha llevado a cabo, como se ha señalado anteriormente, para las 2 actividades libres de la asignatura de Dirección Financiera II de $3 .^{\circ}$ de LADE, que el alumno debe elaborar por bloques de temas. En concreto, y para cada actividad libre, el alumno debe autoevaluar su propia actividad libre (formada por cuestiones, reflexiones, problemas y/o pequeños casos libremente diseñados) y también evaluar de forma anónima la actividad libre de otro compañero. Para ello, los alumnos atenderán a los siguientes aspectos en una escala de 1 a 10: presentación, originalidad, interrelación, relevancia y trabajo (o grado de elaboración).

Además, el profesor evaluará y calificará de forma ciega (esto es, sin conocer los resultados de la autoevaluación y la evaluación por otro compañero) cada actividad libre para, posteriormente, revisar ambas evaluaciones (la autoevaluación y la evaluación por otro compañero) y sus respectivas calificaciones, $y$, sólo si lo considera necesario, por existir una brecha importante (igual o superior a 2 puntos sobre 10), llevará a cabo las oportunas correcciones. En el caso de la evaluación por un compañero, la corrección será la siguiente: (1) si la calificación es al alza, restará al evaluador lo que le ha puesto de más al 
evaluado, y (2) si la calificación es a la baja, sumará al evaluado lo que le ha puesto de menos el evaluador y se lo restará a este último. Las labores de autoevaluación y de evaluación a los compañeros llevadas a cabo correctamente (sin corrección por parte del profesor) sobre las 2 actividades libres se compensarán con 0,1 puntos a sumar a la puntuación obtenida por el evaluador en sus actividades libres tras ponderar por el 15\%, teniendo en cuenta que la máxima calificación que se puede obtener por éstas es de 1,5 puntos, dado que representan el 15\% de la calificación total.

\section{Valoración Por PARTE de LOS ALUMNOS DE LA EVALUACIÓN AUTÓNOMA: AUTOEVALUACIÓN Y EVALUACIÓN POR LOS COMPAÑEROS}

Para ver la valoración que los alumnos otorgan a su participación en la puesta en práctica de esta experiencia de autoevaluación autónoma, al finalizar la misma, se les pasó una encuesta que contenía 8 ítems a cerca de la autoevaluación y otros 8 sobre la evaluación por los compañeros, que debían ser valorados en una escala de 1 a 5. La figura 1 contiene el formulario de la encuesta.

\begin{tabular}{|c|c|c|c|c|c|c|c|c|}
\hline \multicolumn{9}{|c|}{$\begin{array}{l}\text { Encuesta a los estudiantes sobre su participación en la evaluación de las actividades libres a } \\
\qquad y \text { de la evaluación por los compañeros } \\
\text { Responda a los ítems en orden creciente, según este modelo }\end{array}$} \\
\hline 1 & 2 & 3 & 4 & \multicolumn{5}{|c|}{5} \\
\hline $\begin{array}{c}\text { Muy poco } \\
\text { Muy pocas veces }\end{array}$ & $\begin{array}{c}\text { Poco } \\
\text { Pocas veces }\end{array}$ & $\begin{array}{c}\text { Algo } \\
\text { Con frecuencia }\end{array}$ & $\begin{array}{c}\text { Bastante } \\
\text { Casi siempre }\end{array}$ & \multicolumn{5}{|c|}{$\begin{array}{l}\text { Mucho } \\
\text { Siempre }\end{array}$} \\
\hline \multicolumn{4}{|l|}{ Ítems a valorar } & \multicolumn{5}{|c|}{ Indicar con una $X$} \\
\hline & & & & 1 & 2 & 3 & 4 & 5 \\
\hline \multicolumn{4}{|c|}{ Bloque respecto de la autoevaluación } & & & & & \\
\hline \multicolumn{4}{|c|}{ 1. La autoevaluación te ha motivado a trabajar más las actividades libres } & & & & & \\
\hline \multicolumn{4}{|c|}{ 2. Te parece adecuado incorporar en tu trabajo (actividades libres) tu autoevaluación } & & & & & \\
\hline \multicolumn{4}{|c|}{ 3. Es necesario que el profesor supervise tu autoevaluación } & & & & & \\
\hline \multicolumn{4}{|c|}{ 4. Ha sido satisfactoria la experiencia de autoevaluar tu trabajo } & & & & & \\
\hline \multicolumn{4}{|c|}{ 5. Te has tomado en serio esta tarea } & & & & & \\
\hline \multicolumn{4}{|c|}{ 6. Te ha aportado cosas (visión autocrítica, reflexiva, etc.) esta labor de autoevaluación } & & & & & \\
\hline \multicolumn{4}{|c|}{ 7. Crees que has sido responsable y justo en dicha tarea } & & & & & \\
\hline \multicolumn{4}{|c|}{ 8. Consideras que este método de evaluación debe formar parte de los sistemas de evaluación } & & & & & \\
\hline \multicolumn{4}{|c|}{ Bloque respecto de la evaluación por los compañeros } & & & & & \\
\hline \multicolumn{4}{|c|}{ 1. La evaluación por un compañero te ha motivado a trabajar más las actividades libres } & & & & & \\
\hline \multicolumn{4}{|c|}{ 2. Te parece adecuado que un compañero tuyo haya evaluado tu trabajo (actividades libres) } & & & & & \\
\hline \multicolumn{4}{|c|}{ 3. Es necesario que el profesor supervise la evaluación elaborada por un compañero } & & & & & \\
\hline \multicolumn{4}{|c|}{ 4. Ha sido satisfactoria la experiencia de evaluar el trabajo de un compañero } & & & & & \\
\hline \multicolumn{4}{|c|}{ 5. Te has tomado en serio esta tarea } & & & & & \\
\hline \multicolumn{4}{|c|}{ 6. Te ha aportado cosas (nuevos conocimientos, enfoques, visión critica, etc.) esta labor de evaluación } & & & & & \\
\hline \multicolumn{4}{|c|}{ 7. Crees que has sido responsable y justo en dicha tarea } & & & & & \\
\hline \multicolumn{4}{|c|}{ 8. Consideras que este método de evaluación debe formar parte de los sistemas de evaluación } & & & & & \\
\hline
\end{tabular}

Figura 1. Encuesta sobre la valoración que los estudiantes otorgan a su participación en la evaluación autónoma. 
Los resultados de la encuesta para una muestra de 151 alumnos se presentan a continuación, agrupados en autoevaluación, evaluación por los compañeros, y autoevaluación versus evaluación por los compañeros.

\subsection{Evaluación por los compañeros}

La tabla 2 recoge los histogramas de frecuencias de las valoraciones otorgadas por los estudiantes a la evaluación por los compañeros. De nuevo, agrupando las valoraciones negativas ( 1 y 2), por un lado, y las positivas $(3,4$ y 5$)$, por otro, se puede indicar que: al $67 \%$ le ha

La tabla 1 recoge los histogramas de frecuencias de las luación. Agrupando las valoraciones negativas (1 y 2), por un lado, y las positivas $(3,4$ y 5$)$, por otro, se puede señalar que: al 77\% le ha motivado a trabajar más, al $80 \%$ le parece adecuada la autoevaluación, el 85\% desea la supervisión del profesor, al 74\% le ha resultado atractiva la experiencia de autoevaluarse, el $96 \%$ ha sido serio, al 91\% le ha aportado cosas, el $99 \%$ ha sido responsable y justo, y el 70\% considera que debe formar parte del sistema de evaluación.

TABLA 1: HISTOGRAMAS DE FRECUENCIAS SOBRE LA VALORACIÓN OUE LOS ESTUDIANTES OTORGAN A SU PARTICIPACIÓN EN LA AUTOEVALUACIÓN

\begin{tabular}{|c|c|c|c|c|c|c|c|c|}
\hline $\begin{array}{c}\text { İ́ms } \\
\text { Valoración }\end{array}$ & Motivación & Adecuación & Supervisión & Satisfacción & Seriedad & Aportación & Responsab. & Utilización \\
\hline $\mathbf{1}$ & $1,32 \%$ & $7,28 \%$ & $0,00 \%$ & $3,31 \%$ & $0,00 \%$ & $0,00 \%$ & $1,32 \%$ & $8,61 \%$ \\
\hline $\mathbf{2}$ & $21,85 \%$ & $12,58 \%$ & $15,23 \%$ & $23,18 \%$ & $3,97 \%$ & $9,27 \%$ & $0,00 \%$ & $21,85 \%$ \\
\hline $\mathbf{3}$ & $36,42 \%$ & $36,42 \%$ & $7,95 \%$ & $31,79 \%$ & $17,22 \%$ & $39,07 \%$ & $18,54 \%$ & $25,17 \%$ \\
\hline $\mathbf{4}$ & $32,45 \%$ & $25,83 \%$ & $27,81 \%$ & $35,10 \%$ & $43,71 \%$ & $40,40 \%$ & $39,74 \%$ & $21,85 \%$ \\
\hline $\mathbf{5}$ & $7,95 \%$ & $17,88 \%$ & $49,01 \%$ & $6,62 \%$ & $35,10 \%$ & $11,26 \%$ & $40,40 \%$ & $22,52 \%$ \\
\hline
\end{tabular}

TABLA 2: HISTOGRAMAS DE FRECUENCIAS SOBRE LA VALORACIÓN QUE LOS ESTUDIANTES OTORGAN A SU PARTICIPACIÓN EN LA EVALUACIÓN POR LOS COMPAÑEROS

\begin{tabular}{|c|c|c|c|c|c|c|c|c|}
\hline $\begin{array}{c}\text { İtems } \\
\text { Valoración }\end{array}$ & Motivación & Adecuación & Supervisión & Satisfacción & Seriedad & Aportación & Responsab. & Utilización \\
\hline $\mathbf{1}$ & $2,65 \%$ & $7,28 \%$ & $0,00 \%$ & $1,99 \%$ & $0,00 \%$ & $0,66 \%$ & $0,00 \%$ & $7,95 \%$ \\
\hline $\mathbf{2}$ & $30,46 \%$ & $26,49 \%$ & $1,32 \%$ & $9,27 \%$ & $4,64 \%$ & $16,56 \%$ & $2,65 \%$ & $21,85 \%$ \\
\hline $\mathbf{3}$ & $49,01 \%$ & $41,06 \%$ & $6,62 \%$ & $55,63 \%$ & $17,88 \%$ & $32,45 \%$ & $11,26 \%$ & $25,17 \%$ \\
\hline $\mathbf{4}$ & $17,88 \%$ & $20,53 \%$ & $21,19 \%$ & $30,46 \%$ & $32,45 \%$ & $41,72 \%$ & $44,37 \%$ & $33,11 \%$ \\
\hline $\mathbf{5}$ & $0,00 \%$ & $4,64 \%$ & $70,86 \%$ & $2,65 \%$ & $45,03 \%$ & $8,61 \%$ & $41,72 \%$ & $11,92 \%$ \\
\hline
\end{tabular}

motivado a trabajar más, al 66\% le parece adecuada la evaluación por un compañero, el 99\% desea la supervisión del profesor, al $89 \%$ le ha resultado atractiva la experiencia de evaluar, el 95\% ha sido serio, al 83\% le ha aportado cosas, el $97 \%$ ha sido responsable y justo, y el $70 \%$ considera que debe formar parte del sistema de evaluación. 


\subsection{Autoevaluación frente a evaluación por los compañeros}

Enfrentando las valoraciones otorgadas por los estudiantes a la autoevaluación y la evaluación por los compañeros para cada uno de los ítems (véase la tabla 3), mediante los tests de diferencia de medias de muestras relacionadas (tanto paramétricos, t-student, como no paramétricos,
Wilcoxon), se puede señalar que: la autoevaluación les motiva a trabajar más a los alumnos, están más de acuerdo con la autoevaluación que con la evaluación por los compañeros, la supervisión del profesor se demanda más en la evaluación por los compañeros, muy probablemente debido a que se fían poco de la evaluación de sus compañeros, y el resto de variables no presentan diferencias significativas.

TABLA 3: TESTS DE DIFERENCIA DE MEDIAS SOBRE LA VALORACIÓN QUE LOS ESTUDIANTES OTORGAN A SU PARTICIPACIÓN EN LA AUTOEVALUACIÓN FRENTE A LA EVALUACIÓN POR LOS COMPAÑEROS

\begin{tabular}{|c|c|c|c|c|c|c|c|c|}
\hline $\begin{array}{c}\text { İtems } \\
\text { Test }\end{array}$ & Motivación & Adecuación & Supervisión & Satisfacción & Seriedad & Aportación & Responsab. & Utilización \\
\hline t-student & $4,778^{* * *}$ & $4,501^{* * *}$ & $-6,401^{* * *}$ & $-0,513$ & $-1,399$ & 1,458 & $-1,095$ & 1,382 \\
\hline Wilcoxon & $-4,376^{* * *}$ & $-4.166^{* * *}$ & $-5.657^{* * *}$ & $-0,547$ & $-1,342$ & $-1,576$ & $-1,065$ & $-1,534$ \\
\hline
\end{tabular}

** significativo al $5 \%$

*** significativo al $1 \%$.

3.4. Diferencias de calificaciones entre autoevaluación, la evaluación por los compañeros y la evaluación otorgada por el profesor

Por último, enfrentando las calificaciones de la autoevaluación, la evaluación por los compañeros y la evaluación atorgada por el profesor (véase la tabla 4), mediante los tests de diferencia de medias de muestras relacionadas (tanto paramétricos, t-student, como no paramétricos, Wilcoxon), se puede señalar que no existen diferencias significativas entre ellas, a pesar de que los estudiantes se autoevalúan con una calificación por encima de la del profesor entorno a los 0,08 puntos (sobre una calificación total, lógicamente, de 10 puntos) y evalúan a sus compañeros por debajo de la del profesor en torno a los 0,02 puntos. Por tanto, cabe indicar que las calificaciones que los estudiantes establecen en la evaluación autónoma (autoevaluación y evaluación por los compañeros) sobre las actividades libres son bastante objetivas y tan respetables como las otorgadas por el profesor, a tenor de los resultados obtenidos.

TABLA 4: TESTS DE DIFERENCIA DE MEDIAS SOBRE LA CALIFICACIONES DE LA AUTOEVALUACIÓN, LA EVALUACIÓN POR LOS COMPAÑEROS Y LA EVALUACIÓN OTORGADA POR EL PROFESOR

\begin{tabular}{|l|c|c|c|}
\hline \multicolumn{1}{|c|}{ Test } & $\begin{array}{c}\text { Autoevaluación frente a } \\
\text { evaluación por los compañeros }\end{array}$ & $\begin{array}{c}\text { Autoevaluación frente a } \\
\text { evaluación por el profesor }\end{array}$ & $\begin{array}{c}\text { Evaluación por los compañeros } \\
\text { frente a evaluación por el } \\
\text { profesor }\end{array}$ \\
\hline t-student & 1,455 & 1,190 & $-0,337$ \\
\hline Wilcoxon & $-1,227$ & $-0,315$ & $-1,442$ \\
\hline
\end{tabular}

Nótese que las calificaciones de la autoevaluación y la evaluación por los compañeros son previas a las correcciones practicadas por el profesor. 


\section{Conclusiones}

El presente trabajo muestra la puesta en práctica de la evaluación autónoma para las actividades libres de la asignatura de Dirección Financiera II de $3 .^{\circ}$ de LADE y como resultado de la presente experiencia se pretende conocer cómo los alumnos valoran su participación en el proceso de evaluación y qué grado de objetividad presentan las calificaciones obtenidas a través de la autoevaluación y la evaluación por los compañeros frente a las otorgadas por el profesor.

Los resultados revelan que la autoevaluación se valora más por los alumnos que la evaluación por los compañeros en lo relativo a la motivación y adecuación. La supervisión del profesor se demanda más en la evaluación por los compañeros. Ambas experiencias han sido atractivas y han contribuido al desarrollo de los alumnos. Además, se han tomado en serio las tareas de autoevaluación y de evaluación de los compañeros, tratando de ser responsables y justos, y consideran que la autoevaluación y la evaluación por los compañeros deben formar parte de los sistemas de evaluación. Finalmente, no existen diferencias relevantes entre las calificaciones obtenidas en la autoevaluación, la evaluación por los compañeros y la evaluación otorgada por el profesor. En suma, la evaluación autónoma debería ser tenida en cuenta como una técnica e instrumento de evaluación más al servicio de la evaluación del aprendizaje.

\section{BIBLIOGRAFÍA}

Bretones, A. (2008): "Participación del alumnado de Educación Superior en su evaluación", Revista de Educación, vol. 347, pp. 181-202.

Brew, A. (2003): "La autoevalución y la evaluación por los compañeros", en Brown, S. y Glasner, A. (ed.), Evaluar en la Universidad: problemas y nuevos enfoques, Narcea, Madrid, pp. 179189.

Brown, S. (2003): "Estrategia institucionales de evaluación", en Brown, S. y Glasner, A. (ed.), Evaluar en la Universidad: problemas y nuevos enfoques, Narcea, Madrid, pp. 23-33.

Calderón, C. y Escalera, G. (2008): "La evaluación de la docencia ante el reto de Espacio Europeo de Educación Superior (EEES)", Educación XX1, vol. 11, pp. 237-256.

Gessa, A. y Rabadán I. (2009): "La participación del alumno en la evaluación del aprendizaje en la enseñanza superior: una experiencia en estudios empresariales", en Cossío, F. J. (coord.), Administrado entornos inciertos, Congreso Nacional de la Asociación Europea de Dirección y Economía de Empresa, Sevilla.
Gil, J. y Padilla, M. T. (2009): "La participación del alumnado universitario en la evaluación del aprendizaje", Educación $X X 1$, vol. 12, pp. 43-65.

Jordan, S. (2003): "La práctica de la autoevalución y la evaluación por los compañeros", en Brown, S. y Glasner, A. (ed.), Evaluar en la Universidad: Problemas y nuevos enfoques, Narcea, Madrid, pp. 191-201.

Padilla, M. T. y Gil, J. (2008): "La evaluación orientada al aprendizaje en la Educación Superior: Condiciones estratégicas para su aplicación en la enseñanza universitaria", Revista Española de Pedagogía, vol. 241, pp. 467-486.

Pérez, A.; Tabernero, B.; López, V.M.; Ureña, N.; Ruiz, E.; Caplloch, M.; González, N. y Castejón, F. J. (2008): "Evaluación formativa y compartida en la docencia universitaria y el Espacio Europeo de Educación Superior: cuestiones clave para su puesta en práctica", Revista de Educación, vol. 347, pp. 435-451.

Webster, R. y Lapaham, A. (2003): "Evaluación realizada por los compañeros: motivaciones, reflexiones y perspectiva de futuro", en Brown, S. y Glasner, A. (ed.), Evaluar en la Universidad: problemas y nuevos enfoques, Narcea, Madrid, pp. 203-210.

doi: 10.3989/arbor.2011.Extra-3n3142 